\title{
Selected factors influencing wear comfort of clothing: case studies
}

\begin{abstract}
Wear comfort is a fundamental and universal need for consumers and depends on a multitude of factors among which thermo-physiological comfort, skin sensorial comfort and garment construction parameters such as design and fit. This paper discusses three case-studies which demonstrate the importance of the design, fit and fabric finish on wear comfort. The studies involved:

a. The application of phase change materials (PCMs) to the fabric used in unisuits for rowers to better maintain their thermal balance during exercise.

b. Improving the design of workwear for nursing staff.

c. The development of well fitted functional body wear involving moisture regulating fabrics to prevent non-aesthetic sweat stains on shirts and blouses.

The results have shown that minor adaptations to the design and fit which is well adjusted to the body shape of the wearer can have a major impact on the thermophysiological and tactile comfort feeling of the wearer. On the other hand, the use of phase change materials had little effect on the skin temperature of the wearer which was in stark contrast with the use of moisture regulating fabrics that indeed largely influence overall comfort sensation of garments. These insights were very valuable to the garment manufacturers which meanwhile have implemented these results into their products. The adapted nursing uniforms were taken into production and enthusiastically welcomed by the end users. Shortly the functional body wear will also come available on the market.
\end{abstract}

Keywords: wear comfort, fit, clothing design, pcms finishing
Volume 4 Issue I - 2018

\author{
Alexandra De Raeve, Simona Vasile, Joris \\ Cools \\ Faculty of Science and Technology, Department of Textiles, \\ University College Ghent, Belgium
}

\begin{abstract}
Correspondence: Alexandra De Raeve, Faculty of Science and Technology, Department of Textiles, Fashion and Wood technologies, University College Ghent, Buchtenstraat II, 905 I Sint-Denijs-Westrem, Belgium, Tel 3292432736, Email Alexandra.deraeve@hogent.be
\end{abstract}

Received: January 22, 2018 | Published: February 06, 2018

\section{Introduction}

Comfort is a perception of well-being in which the human body is in psychological, physiological and physical harmony with the environment where it is situated. Clothing is an integrated part of this. The physiological and psychological conditions include a number of issues such as:

i. Thermo-physiological comfort: achieving a comfortable thermal and humid condition in which the thermo-regulating capacity, the thermal insulation and the moisture-regulating capacity of the garment are considered as a function of environmental conditions (climate) and the working conditions (level of effort). Thermo-physiological comfort is strongly affected by the fabric, the style and the fit.

ii. Skin sensorial comfort: the induction of various nerve impulses when the textile is in contact with the skin in which the softness, mobility, skin tolerance and adhesion effects are considered. Sensorial comfort is strongly affected by the fabric, the fit and the assembly technique.

iii. Ability to move, which is mainly affected by the fit and ease allowance.

iv. Aesthetics: the subjective perception of a garment that contributes to the overall sense of well- being which is in relation with the design, the fit and the overall quality of the garment.

Scientists agree however that the thermo-regulating and moistureregulating properties of a garment are the main factors in comfort as it is of vital importance that the heat generation and heat loss of man is in balance over a long term (heat generation=heat loss). ${ }^{1}$ The main four factors affect heat balance of man: the climate, the clothing, the effort level and individual factors. Usually the body temperature is above the ambient temperature. An inside source is necessary to provide the necessary heat to maintain our body temperature. This is ensured by the metabolism. Of the energy absorbed by nutrition, 15$30 \%$ is converted into useful work and 70 to $85 \%$ is released in the form of heat. Physical work also contributes to a large extent to the generation of heat. Any activity above what is necessary to maintain the body temperature constant, results in an excess of heat that must be dissipated. A lower activity results in cooling when the body is insufficiently isolated. High ambient temperatures make it difficult to dispose sufficiently of excess heat. When an imbalance occurs between the heat produced in the body and the release of heat by the body, the body temperature increases and we speak of hyperthermia. This can result in various health problems from cramps to a cardiac arrest. In extremely cold conditions, the body will lose more heat than it can produce. In this case we speak of hypothermia. This may lead to freezing of the extremities. In addition the safety of workers may come at risk.

The human body has four mechanisms (i.e. conduction, convection, radiation, evaporation) to discharge excess heat to the environment in order to maintain thermal equilibrium. ${ }^{2}$ Partition of the mechanisms is a function of environmental parameters. Heat loss by conduction occurs when the body is in direct contact with another substance (air, water...). The ratio depends on the temperature difference between the two substances. For instance, one such body 
loses heat when immersed in cold water. In the case of convection, heat is removed by a moving substance (liquid or gas). In high winds, more heat is removed by convection. In the event of radiation heat transfer occurs by electromagnetic radiation. Rays give little heat as they go by air, but once they hit a body, their energy is converted into heat. There is usually little heat loss by radiation as it heavily depends on the temperature of the body. On the contrary, radiation can be a major source of heat absorption coming from hot bodies such as the sun, radiators or a fire. Radiation and absorption are also affected by the colour of the body. Clothing decreases the loss by radiation by reducing the temperature difference between the body and the environment. The clothing is in fact the immediate vicinity of the person. Evaporation is a highly efficient cooling mechanism. Turning water into vapour requires very large amounts of energy. The evaporation of $1 \mathrm{~g}$ of water at body core temperature $\left(37^{\circ} \mathrm{C}\right)$ requires $2424 \mathrm{~J}$. When water evaporates on the skin, the necessary energy is extracted from the skin which cools the body severely. When the ambient temperature approaches the body temperature, heat loss through convection and radiation stops remaining only evaporation (sweating). This becomes difficult in tropical conditions.

Clothing has a major impact on the comfort feeling of people. The clothing properties that will affect this comfort feeling are the thermal conductivity, the moisture and vapour transport capacity, the air permeability, the fit and the touch and feel. The thermal resistance or obstruction of heat exchange by convection, conduction and radiation is by $20 \%$ determined by fiber-related factors (chemistry and morphology, fineness and crimp, density, distribution) and by $80 \%$ by construction-related parameters. ${ }^{3}$ Here we consider both the textile material, (i.e. voluminousity of the fabric and its finishing or coating) and the technical components of the garment construction. Thermal insulation of a garment is strongly affected by the layers of air present in and between the textile material and the wearer's body. These are largely determined by the design and fit. Integrating an open structure (e.g. mesh) can cause a turbulent air flow which can increase the heat dissipation by convection. The resulting isolation of a garment reduces by $20 \%$ in a sitting position and by $50 \%$ during cycling and walking. In combination with wind and rain this may even reach $90 \%{ }^{3}$ The moisture regulating ability of a garment is determined by the textile material. This involves the wicking ability, absorbency and water vapour permeability. Also, air layers within the garment will affect moisture transportation. In clothing which aims at being a barrier against water, chemicals, gases, blood, etc. sweat is hard to evaporate and therefore the body cannot cool down easily. The most powerful cooling system, sweating is thus put out of play. The local insertion of openings or open structures in the design can facilitate the evaporation of sweat.

\section{Materials and methods}

The following three cases will investigate the effect of material and clothing properties on the overall comfort feeling of the wearer.

The first case study examined the effect of phase change materials (PCMs) on the thermo-regulating capacity of unisuits for rowers. PCMs are substances with a high enthalpy of fusion making them capable of storing or releasing a large amount of energy when switching from the solid state to the liquid state and vice versa. Garments containing PCMs immediately react to environmental temperature changes ensuring thermal protection of the body by maintaining the heat balance. ${ }^{4}$ five highly trained rowers (i.e. 3 males and 2 females), aged between 13 and 18 years performed an indoor ergometer test with a total duration of nine minutes. The room temperature and relative humidity during the test varied between $20.2^{\circ} \mathrm{C}-20.9^{\circ} \mathrm{C}$ and between RH $60 \%-67 \%$, respectively. The air speed was not controlled, but the athletes were subject to a constant air flow coming from the ergometer flywheel. The test was interrupted every 3 minutes to take a picture with a TESTO thermal imaging camera ${ }^{5}$ to assess the torso's skin temperature distribution. The rowers wore a unisuit treated with $10 \%$ owf of PCMs (Thermic ${ }^{\mathrm{TM}}$ from Devan Chemicals). The PCMs were added to the unisuits by soaking them in a solution comprising PCM microcapsules followed by curing at $140^{\circ} \mathrm{C}$. Thermic ${ }^{\mathrm{TM}} \mathrm{PCMs}$ are based on paraffine (octadecane) with a melting temperature of $28^{\circ} \mathrm{C}$. After cooling down the test was repeated with an untreated unisuit.

After exercising the rowers were questioned about their overall comfort sensation on a scale of 1 to 4 . The thermal resistance $\left(R_{\mathrm{es}}\right)$ and resistance to evaporation $\left(\mathrm{R}_{\mathrm{et}}\right)$ of both the treated and untreated unisuits were measured on a Newton sweating thermal manikin (Figure 1) in static and dynamic conditions (manikin simulating walking at a speed of $3.5 \mathrm{~km} / \mathrm{h}$ ) according to ISO $15831 .{ }^{6}$ The results were compared with the wear trials: outdoor training with duration of 90minutes, at a temperature of $22^{\circ} \mathrm{C}$, partially clouded. It was hypothesed that the mean and local skin temperature of subjects wearing PCMs-treated unisuits will be lower during the whole 9-minutes test protocol.

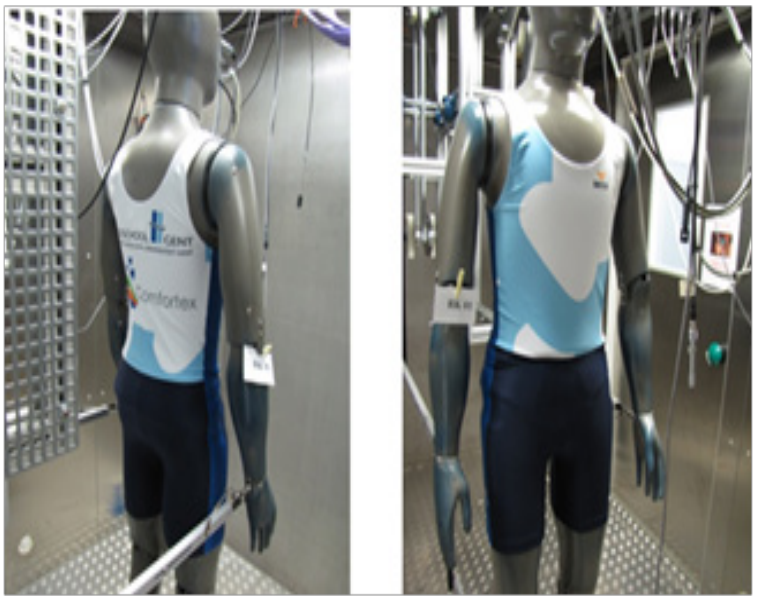

Figure I Testing of unisuit on Newton sweating thermal manikin.

Due to the relatively high temperature of the working environment $\left(22^{\circ} \mathrm{C}-30^{\circ} \mathrm{C}\right)$, excessive sweating and overheating is a common problem with nursing staff. This was confirmed in a survey with 21 nursing students expressing their needs and comfort feeling in their standard ensemble. The questionnaire revealed that all participants sweat while doing their work. Excessive sweating mainly aroused on the back and arm pits. Over $60 \%$ of subjects said this makes them feel uncomfortable (Figure 2).

Based on the results of the survey a new design was developed aiming at improved comfort. This second case study examined the effect of garment fit and the insertion of ventilation openings (mesh fabric) at places on the body where high sweat production occurs in nursing vests (back, arm pits, upper arms and shoulders) on the overall comfort feeling of nursing staff. The newly designed ensemble (Figure 3 ) was made in the same fabric as the standard unisex ensemble, a $65 / 35$ polyester/cotton blend with a weight of $215 \mathrm{~g} / \mathrm{m}^{2}$. At the level of the shoulders, back, upper arms and arm pits a $100 \%$ polyester 
mesh fabric of $115 \mathrm{~g} / \mathrm{m}^{2}$ was integrated. Wear trials were performed over a period of 15 days. The test subjects wore the same sub layers as with their standard ensemble. In order to measure the thermal resistance and resistance to evaporation, both the standard and newly designed ensembles were tested on the Newton sweating thermal manikin (Figure 4) in static and dynamic conditions according to ISO 15831(ISO, 2004).

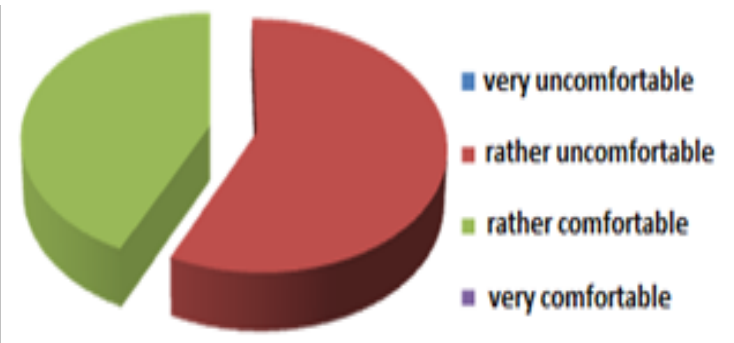

Figure 2 Comfort feeling in standard nursing ensemble.

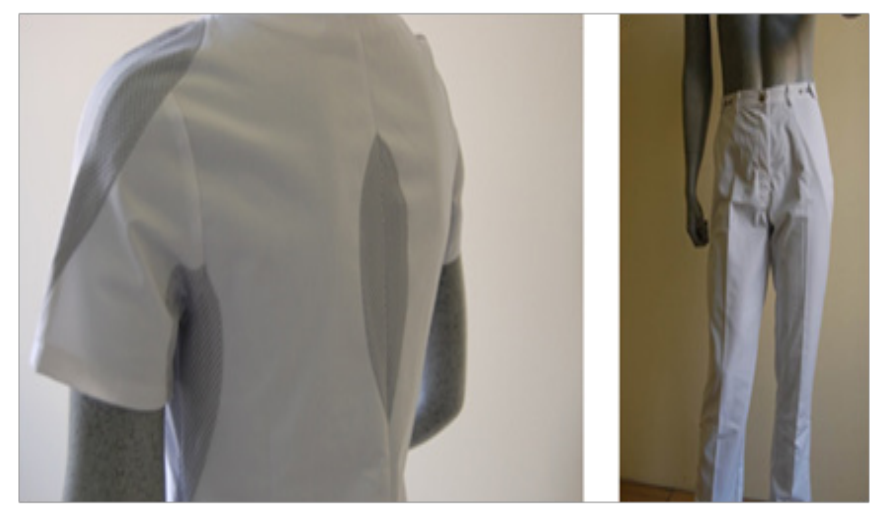

Figure $3 \mathrm{New}$ design of nursing vest and trousers.
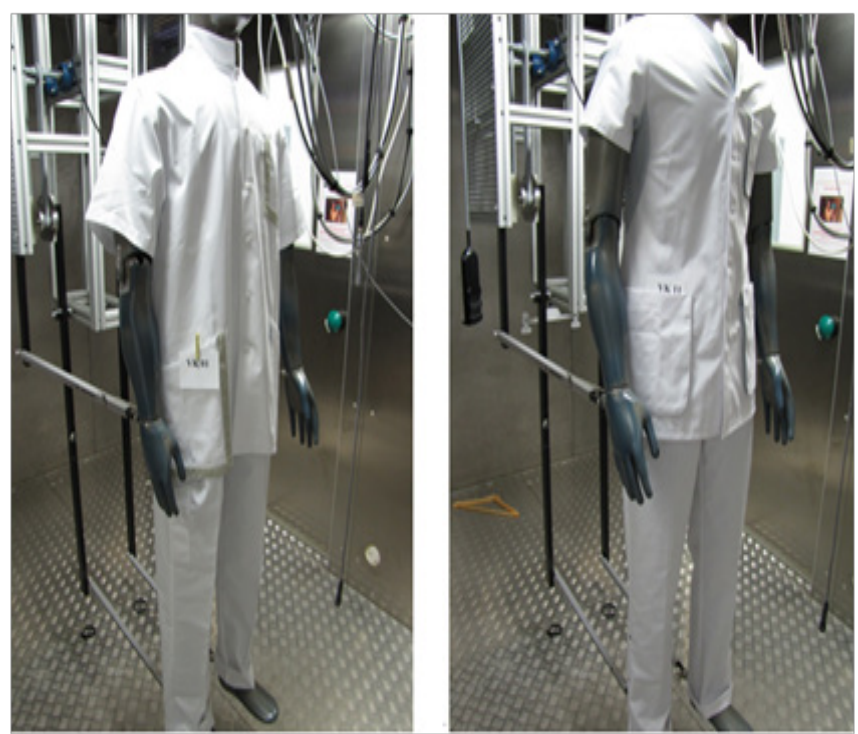

Figure 4 Testing of standard ensemble (left) and newly designed ensemble (right) on Newton sweating thermal manikin.

The third case involved the development of moisture regulating body wear to prevent sweat stains. About $60 \%$ of 228 subjects, age $18-55$ (76\% between $18-25$ year) participating in a survey complained about excessive sweating at the level of the armpits, lower back, shoulders (32\% for males) and chest/ breasts area ( $24 \%$ of females).
Approximatively half of the male and female subjects coped with visible sweat stains. The majority of the male $(63 \%)$ and females (52\%) interviewed normally don't wear body wear but $75 \%$ were potentially interested in wearing a fitted model (68\% male versus $91 \%$ female), with a V-neckline ( $62 \%$ of females) or round neckline $(53 \%$ males). Several body wear models were developed by alternating fit, neckline, and fabric. Figure 5 shows three models A-C of body wear for females with round and V-neckline, developed with knitted fabric of comparable weight per unit area $\left(150 \mathrm{~g} / \mathrm{m}^{2}\right)$ but different composition: 90/10 Polyamide/ Elastane (fabric A) and micro modal fibers (fabric B).

The male models were similar but looser at waist level. Physical properties of fabrics A and B were measured by means of a Fabric Touch Tester (FTT) which subsequently predicts fabric sensorial properties (i.e. smoothness, softness, warmth, total active and passive touch feel). ${ }^{7}$ Presence of sweat stains, fit, thermal and sensorial comfort of the body wear A-C was evaluated by four men (mean age 39 year) and four women (mean age 20year). Except one case, the T-shirts were worn under one layer clothing with short-sleeves (i.e. shirt, polo) or long-sleeves blouse or shirt. They were worn during various activities (i.e. light-intensity deskwork, walking, cycling, jogging) performed at temperature between $19-28^{\circ} \mathrm{C}$. Overall comfort sensation was evaluated on a scale of 1 to 5 with a score 1 meaning very uncomfortable and score 5 very comfortable.

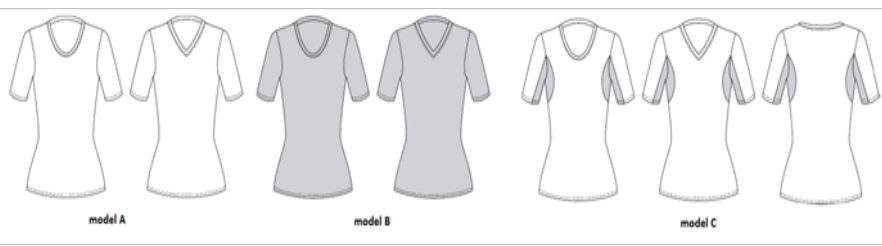

Figure 5 Female body wear (model A- B- C) in fabric A (white) or B (black) with round or V-neckline.

\section{Results}

In the first case the effect of PCMs on the thermo-regulating capacity of unisuits for rowers was examined. The software of the TESTO thermal imaging camera calculated the average skin temperature of the torso which is depicted in Table 1 for each of the five subjects wearing a treated or a control unisuit. An example of the distribution of the skin temperature of subject 1 prior to exercising is shown in Figure 6. Due to the evaporation of sweat and the cooling effect of the air flow coming from the flywheel of the ergometer the skin temperature of the torso decreased during exercising. During the first 3 minutes of the test, the temperature decrease is more rapid in the treated suits. In the second stage of the test the effect of air flow and sweat on the decrease of the skin temperature is more noticeable in the untreated suits. In the cool down phase, the average skin temperature of the torso rose again. At the end of the test (3minutes after stopping the exercise) the skin temperature of three out of five subjects wearing a treated suit was higher.

As depicted in Figure 7, the manikin tests showed that the treated unisuits have a slightly higher thermal resistance $\left(\mathrm{R}_{\mathrm{ct}}\right)$ on the stomach, back and hip front in static conditions and on the stomach and back in dynamic conditions. The resistance to evaporation $\left(\mathrm{R}_{\mathrm{ee}}\right)$ of the PCMtreated unisuit was, in both conditions generally lower (Figure 8) as compared to the control unisuit, which suggests that this unisuit will allow more sweat evaporation. 
Table I Average skin temperature of the test persons (I-5) wearing a control suit (untreated) or a PCM-treated unisuit (I0\% Thermic ${ }^{\mathrm{TM}}$ ) during exercise (T0-T9min) and during recovery (TI2min).

\begin{tabular}{|c|c|c|c|c|c|c|}
\hline Subject & Unisuit & $\mathrm{T} 0 \mathrm{~min}\left({ }^{\circ} \mathrm{C}\right)$ & $\mathrm{T} 3 \mathrm{~min}\left({ }^{\circ} \mathrm{C}\right)$ & $\mathrm{T} 6 \mathrm{~min}\left({ }^{\circ} \mathrm{C}\right)$ & $\mathrm{T} 9 \min \left({ }^{\circ} \mathrm{C}\right)$ & $\mathrm{T} / 2 \mathrm{~min}\left({ }^{\circ} \mathrm{C}\right)$ \\
\hline \multirow[t]{2}{*}{1} & Untreated & 28.2 & 28 & 27.3 & 26.9 & 28 \\
\hline & I0 \% Thermic ${ }^{\mathrm{TM}}$ & 29.6 & 27.8 & 27.2 & 27.2 & 27.9 \\
\hline \multirow[t]{2}{*}{2} & untreated & 30.9 & 29.3 & 29.2 & 29.1 & 29.9 \\
\hline & $10 \%$ Thermic $^{\mathrm{TM}}$ & 31.3 & 29.5 & 28.6 & 28.8 & 30 \\
\hline \multirow[t]{2}{*}{3} & untreated & 28.6 & 27.9 & 27.5 & 27.1 & 27.9 \\
\hline & $10 \%$ Thermic ${ }^{\mathrm{TM}}$ & 30 & 28.5 & 27.8 & 27.6 & 28.7 \\
\hline \multirow[t]{2}{*}{4} & untreated & 28.9 & 28.5 & 27.3 & 27.1 & 28.5 \\
\hline & $10 \%$ Thermic $^{\mathrm{TM}}$ & 30.6 & 29.5 & 28.1 & 27.2 & 29.3 \\
\hline \multirow[t]{2}{*}{5} & untreated & 30.5 & 28.9 & 28.3 & 28.8 & 30.7 \\
\hline & $10 \%$ Thermic $^{\mathrm{TM}}$ & 31.3 & 29.9 & 29 & 28.8 & 30.3 \\
\hline
\end{tabular}

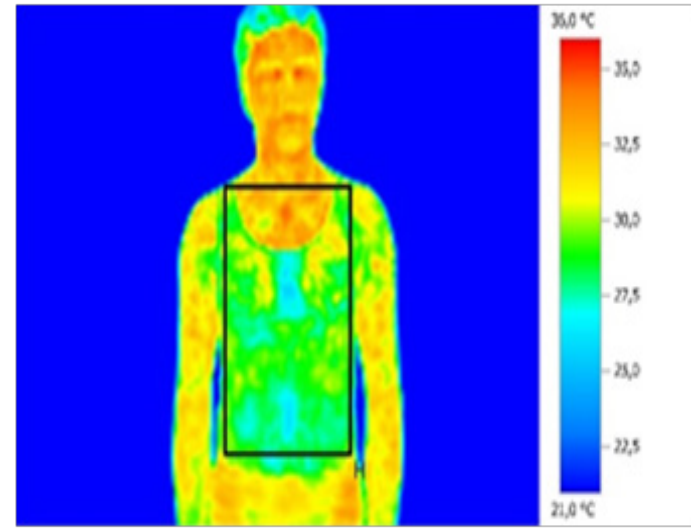

Figure 6 Thermal image of torso temperature of subject I prior to exercise.

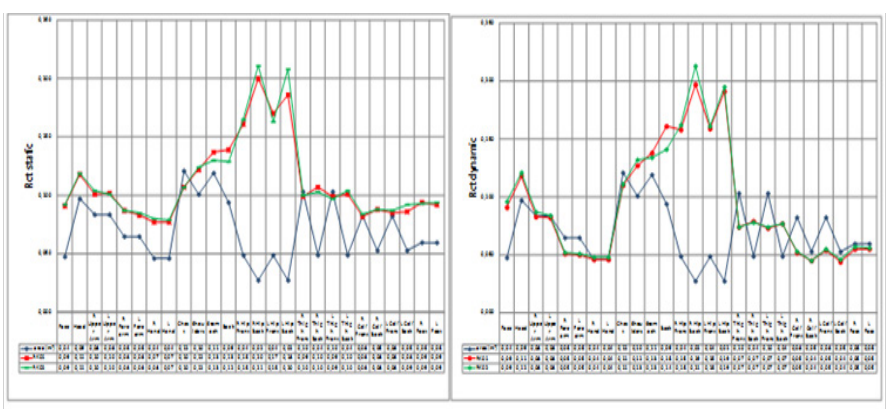

Figure 7 Thermal resistance Rct of untreated (red) and treated (green) unisuits, at various body locations, in static (left) and dynamic (right) conditions.

The test subjects involved in the wear trials indicated they felt less hot and clammy and thus more comfortable (scale 3-4) after exercising in the treated unisuits as compared to the untreated suits (scale 2-3). This seems to be in some agreement with the results from the manikin.

In the second case which evaluated the effect of a new design in nursing ensembles over $75 \%$ of the test subjects indicated they felt more comfortable in the newly designed ensembles (Figure 9). That indicating wear comfort could still be improved complained about the high weight of the fabric. Some male test subjects found the design too feminine.

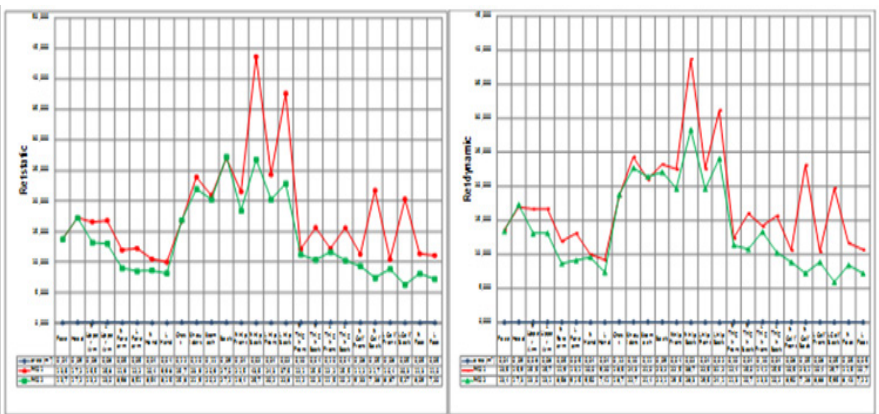

Figure 8 Resistance to evaporation Ret of untreated (red) and treated (green) unisuits, at various body locations, in static (left) and dynamic (right) conditions.

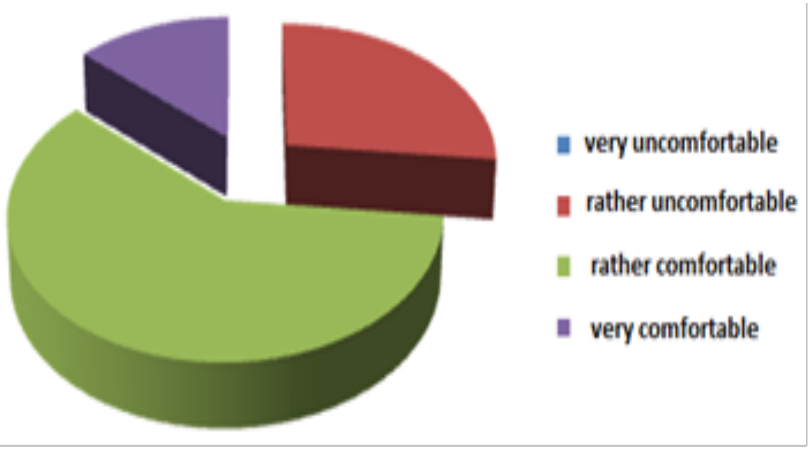

Figure 9 Comfort feeling in newly designed nursing ensemble.

The manikin tests showed that thermal resistance $\left(\mathrm{R}_{\mathrm{ct}}\right)$ of the newly designed ensembles is lower, all over the body, when tested in static conditions. In dynamic conditions the difference is smaller, but still noticeable. Positive effect of the polyester mesh, was observed, especially in static conditions, at the level of upper arm and also slightly on the back, as shown by lower $\mathrm{R}_{\mathrm{ct}}$ (Figure 10). The resistance to evaporation $\left(\mathrm{R}_{\mathrm{et}}\right)$ was also reduced in the new design, on most body areas, both in static and dynamic conditions. Unexpectedly, in static conditions, Ret of the new suit exhibited slightly higher values on the back (Figure 11).

The positive effect of the adapted fit and integration of ventilation openings on the thermo- and moisture regulating abilities of the modified nursing ensemble shown on the sweating thermal manikin is confirmed by the wear trials. 
The third case investigated the influence of fabric, fit and design on comfort of a body wear. Similar sensorial comfort (i.e. touch feel) was predicted by the FTT equipment for fabric A and B. For active touch feel, polyamide fabric A was quoted unexpectedly as slightly softer (grade 4) than modal fabric B (grade 3), as shown in Figure 12 left. Smoothness and warmth of both fabrics were similar, both for active and passive touch feel (Figure 12 right). Active and passive touch feel refers to dynamic evaluation (with the fingers) and during wearing respectively.

Model A and B scored similarly and better than model B before and during performing an activity but all three felt/ scored equally at the end of activity. Unlike models A and C, assessed as wet and clammy by some subjects, model B was found as "neutral", although FTT indicated fabric A and B similar with respect of warmth. Except some complains related to fit and sweat stains at level of armpit in half of the cases, model B based in micromodal fibers was most preferred.

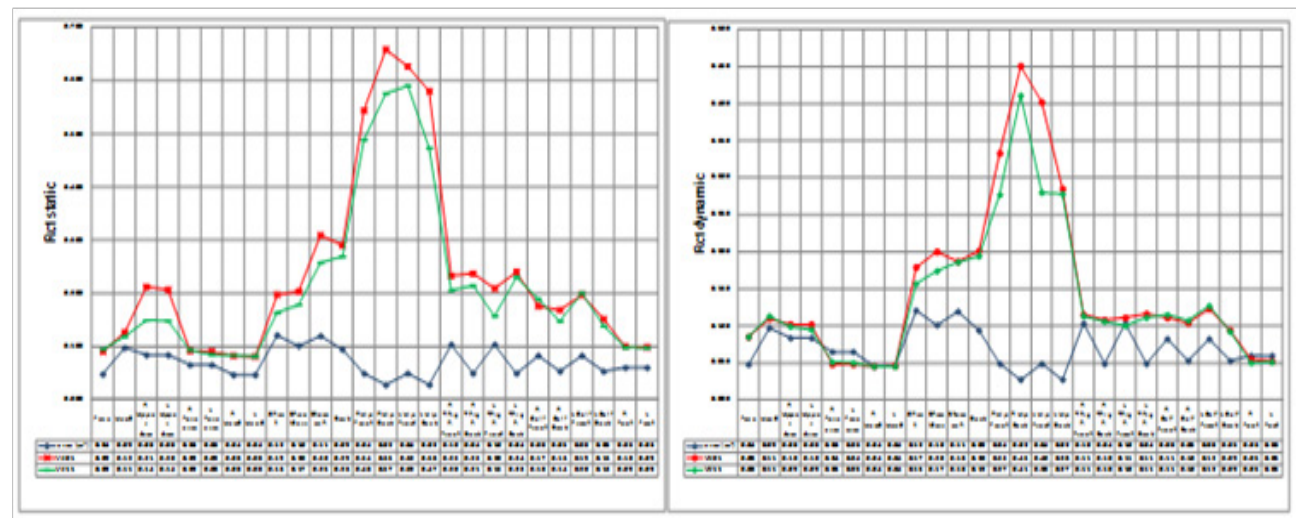

Figure 10 Thermal resistances (Rct) of the standard ensemble (red) and the new design (green), at various body locations, -in static (left) and dynamic (right) conditions.

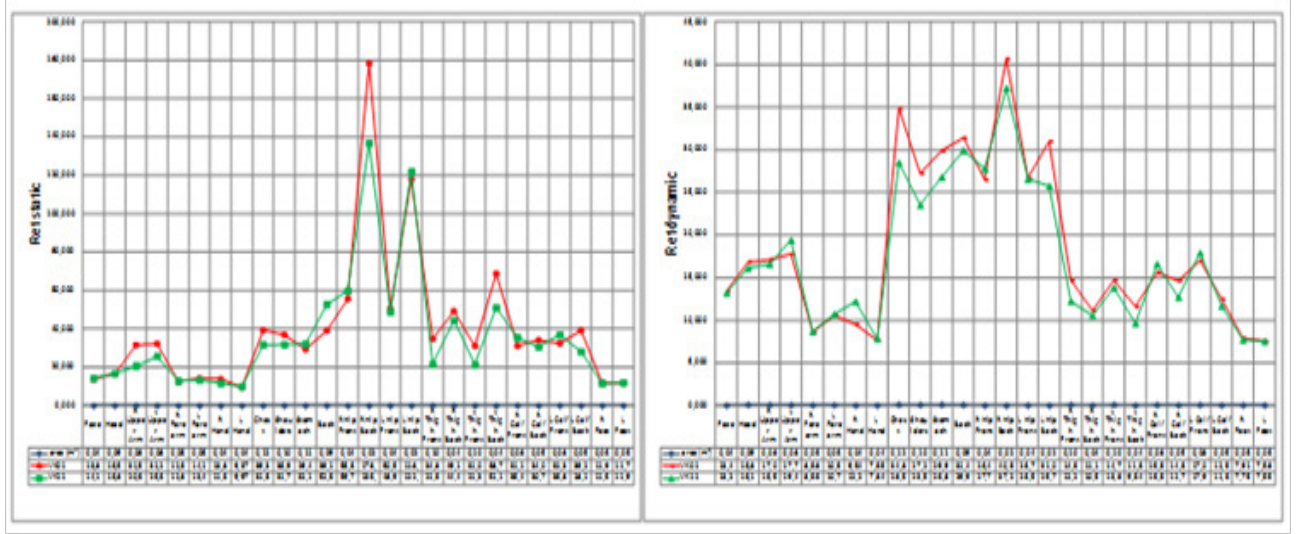

Figure II Resistance to evaporation (Ret) of the standard ensemble (red) and the new design (green), at various body locations, -in static (left) and dynamic (right) conditions.
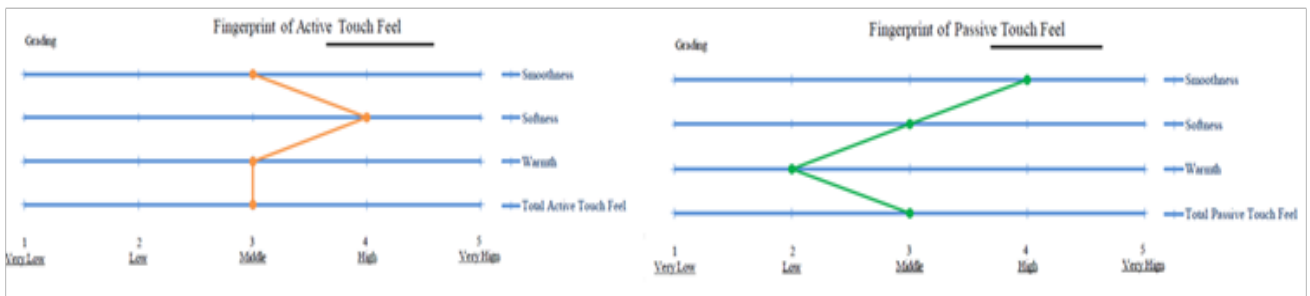

Figure 12 Fingerprint of active touch feels for polyamide fabric $A$ (left) and identical passive touch feel of fabric $A$ and $B$ (right).

\section{Conclusion}

The more rapid temperature decrease in the treated rower unisuits during the first 3 minutes of the test can be explained by the cooling effect of the melting PCMs. The stronger cooling effect of the air flow and sweat on the skin temperature while wearing an untreated suit can be explained by the temperature stabilizing effect of the PCMs in this stage of the test. Although small, this effect remained noticeable during recovery time. Nevertheless, the results of the thermal imaging camera showed little differences between the treated and untreated unisuits. PCMs melt under influence of excess body heat. We may conclude that in this short period of the ergometer test $(9 \mathrm{~min})$ not all PCMs will have been melted and that the effectiveness of PCM treated garment is proportional to the amount of PCMs available for melting during exercise. During the wear trials of 90minutes the PCMs could fully deploy their thermo-regulating effect. This explains that 3 out of 5 test subjects indicated that after training for 90 minutes they felt much more comfortable in the treated unisuits.

The positive results of the newly designed nursing ensemble may 
be explained as follows. The standard nursing ensemble is a loose fit whereas the new design is tight fitted. Body movements generate turbulent air flows in loose fitting garments which subsequently lower the thermal resistance and generate a pumping effect. Adding vents allows a tighter fit with improved heat dissipation. This tight fit allows a more efficient spreading of excessive liquid sweat which, as a consequence, will improve evaporation. On the other hand, the excessive sweat in vapour phase is easier evaporated through the vents. Meanwhile, this new design has been successfully introduced into the market.

In general, the fit of all three models body wear A-C should be improved by adjusting neckline, length and width of sleeves for female-model and length for male-model respectively. Despite of the limited number of subjects and wear protocol limitations (i.e. differences in clothing layers, activity and environmental conditions), this case study gives already a good idea about the complex materialfit-design interaction that leads to different human perception with respect to thermo-physiological and sensorial comfort.

\section{Acknowledgments}

This research was funded in part through a grant by VLAIO. Furthermore the authors wish to thank Dr. Jean Leonard of Centexbel for his help and efforts completing the manikin tests.

\section{Conflict of interest}

Author declares there is no conflict of interest.

\section{References}

1. Daanen HA. Warm aanbevolen. 2004.

2. Saville B. Physical Testing of Textiles. Cambridge: Woodhead Publishing; 1999. p. 336.

3. Van Parys M. Textiel en Comfort. 1994. p. 4-11.

4. Mondal S. Thermo-regulating textiles with phase-change materials. In: Functional textiles for improved performance, protection and health. Woodhead Publishing; 2011. p. 163-183.

5. Testo. Thermal imaging cameras. Testo SE \& Co, UK; 2017.

6. ISO. Clothing-Physiological effects-Measurement of thermal insulationby means of thermal manikin. International Organization for Standardization, Geneva, Switzerland; 2004.

7. Fabric Touch Tester 